\title{
Simulação de Reservatórios de Óleo Leve com Poço Horizontal Hidraulicamente Fraturado
}

\author{
Camila Lima Chung \\ Engenharia Mecânica, Instituto Politécnico, UERJ, Brasil. \\ Grazione de Souza \\ Departamento de Modelagem Computacional, Instituto Politécnico, UERJ, \\ Brasil. \\ Helio Pedro Amaral Souto \\ Departamento de Modelagem Computacional, Instituto Politécnico, UERJ, \\ Brasil.
}

\begin{abstract}
Since the 1960s, because of the relevance to the oil industry, the numerical simulation of hydrocarbon reservoirs has received special attention and has been the subject of extensive studies. The main goal of computational modeling and the use of numerical methods for reservoir simulation is to allow better placement and control of wells, so that there is a optimized oil recovery. In this work, production of hydraulically fractured horizontal wells in light tight oil reservoirs will be studied. In this case, fractures do not form a continuous conductive network and can communicate hydraulically with only the horizontal producer well. In order to do that, a simulator for three-dimensional oil flow in reservoirs, suitable for applications in the field scale, already developed, using the Cartesian coordinate system and a finite difference approach, will be applied for the study of hydraulically fractured horizontal wells. Originally, this simulator and its grid refinement tools had been used only on the simulation of naturally fractured reservoirs. The nonlinear partial differential equation resulting from physical-mathematical modeling, written in terms of pressure, will be solved numerically after discretization and linearization using the Preconditioned Conjugate Gradient method. The main objective is to study the combined effects of hydraulic fractures and horizontal well on the wellbore pressure profile, considering different light tight oil production scenarios. Numerical simulations displayed the influence of important parameters on the well-reservoir system in study, such as fracture permeability and matrix porosity. A study of this type is relevant on the discussion of reservoir production strategies, helping on the decisions about a hydraulic fracturing operation in order to obtain economic viability for the hydrocarbons recovery project.
\end{abstract}

Keywords: reservoir simulation, light tight oil, horizontal well. hydraulic fracturing, finite diferences method.

Corresponding author: Helio Pedro Amaral Souto, helio@iprj.uerj.br

Received: 10 May 2017 / Accepted: 04 July 2017 / Published: 27 Aug 2017.

\section{Introdução}

Desde a década de 1960, devido à pertinência para a indústria petrolífera, a simulação numérica de reservatórios portadores de hidrocarbonetos vem recebendo uma atenção especial e tem sido alvo de amplos estudos. Do ponto de vista econômico, o conhecimento do comportamento do escoamento no reservatório durante a sua explotação é relevante, porque ele fornece dados que ajudam a estimar as características da produção de petróleo,

CALIBRE - Revista Brasiliense de Engenharia e Física Aplicada - Copyright @ 2017, Faculdades ICESP padrões de fluxo, pressão no poço e tempo de produção, por exemplo. O principal objetivo da modelagem computacional e do emprego de métodos numéricos, para a simulação de reservatórios de petróleo, é o de possibilitar um melhor posicionamento e controle dos poços, de forma que haja um aumento no fator de recuperação de hidrocarbonetos.

Dentre os problemas que suscitam um grande interesse encontra-se o fraturamento hidráulico em reservatórios com poços horizontais de produção. Esses dois elementos podem levar ao desenvolvimento de vários regimes de escoamento linear em reservatórios com poro- 
sidade dupla [1]. Entender a influência desses dois mecanismos na produtividade dos reservatórios é crucial para a indústria do petróleo. Muitos autores têm estudado e desenvolvido modelos para a simulação de poços horizontais com fraturamento hidráulico, como por exemplo, [2], [3], [4], [5] e [6], incluindo a aplicação em reservatórios de baixa permeabilidade portadores de óleo leve ([7] e [8]).

\subsection{Poço horizontal}

A utilização de poços horizontais na explotação de reservatórios de petróleo é cada vez mais intensa. Devido à característica dos reservatórios possuírem pequena espessura e grande extensão, os poços horizontais tem diversas vantagens quando comparados aos poços verticais, especialmente por possuírem uma maior área de contato entre o poço e o reservatório.

Os poços horizontais são paralelos ao acamadamento principal do reservatório (Fig. 1) e geralmente são utilizados em casos tais como: reservatórios inclinados, reservatórios depletados, formação com várias camadas e reservatórios nos quais técnicas convencionais tem baixo desempenho ou até mesmo falham. Alguns exemplos são os reservatórios de baixa espessura, com tendência à formação de cones de gás e/ou água, naturalmente fraturados, de baixa permeabilidade e alta anisotropia ou reservatórios com baixa eficiência de varrido [9].

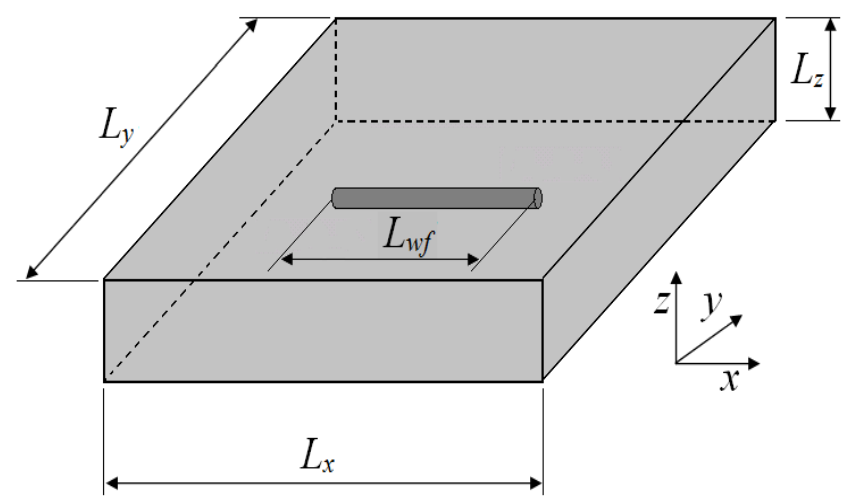

Figura 1: Ilustração de reservatório com poço de produção horizontal.

A variação da pressão por metro no poço horizontal é menor que no poço vertical, quando comparados para a mesma vazão de fluxo. Isso torna o poço horizontal mais atrativo em aplicações de recuperação de óleo e em situações onde a presença de um cone de água é um problema. Dessa forma, os poços horizontais são considerados excelentes ferramentas para a produção do reservatório. Com relação a aplicações de poços horizontais, geralmente considera-se duas categorias: resolução de problemas decorrentes das heterogeneidades do reservatório e resolução de problemas decorrentes das características do escoamento dos fluidos. Ambas aplicações podem estar presentes no mesmo sistema [10].
Destaca-se que a perfuração horizontal pode ser aplicada em qualquer fase de recuperação de um reservatório. Além disso, é sabido que os poços horizontais contribuem significativamente para o aumento da produtividade dos reservatórios com baixa permeabilidade, que podem não produzir sem o emprego de técnicas de estimulação como, por exemplo, o fraturamento hidráulico.

\subsection{Fraturamento hidráulico}

Em formações de baixa permeabilidade o emprego de estimulação é necessário, uma vez que o poço já tenha sido perfurado. O tipo mais comum de estimulação utilizado na indústria de óleo e gás é conhecido como fraturamento hidráulico ou fracking. Aplicando alta pressão por bombeamento de fluidos dentro do poço, são criados caminhos no reservatório por onde o óleo possa fluir para o poço [7].

A operação de fraturamento hidráulico consiste na aplicação de um diferencial de pressão, que provoca quebra da formação (fratura). Também há a injeção de um agente de sustentação (propantes). Esse agente de sustentação, que pode ser à base de areia ou outros compostos, evita o fechamento da fratura (devido às altas pressões existentes nesses tipos de reservatórios) após a retirada da pressão imposta pelo bombeamento dos fluidos. Desta forma, são criados caminhos preferenciais de elevada condutividade que facilitarão o escoamento dos fluidos do reservatório para o poço, ou vice-versa [11].

A permeabilidade em reservatórios convencionais é suficiente para que o fraturamento hidráulico não seja necessário para o aumento da taxa de produção. Por outro lado, a permeabilidade nos reservatórios não convencionais é geralmente baixa, de modo que as fraturas hidráulicas são criadas para permitir o aumento da produção economicamente viável de óleo e gás [7]. A Fig. 2 traz uma ilustração de um poço horizontal hidraulicamente fraturado.

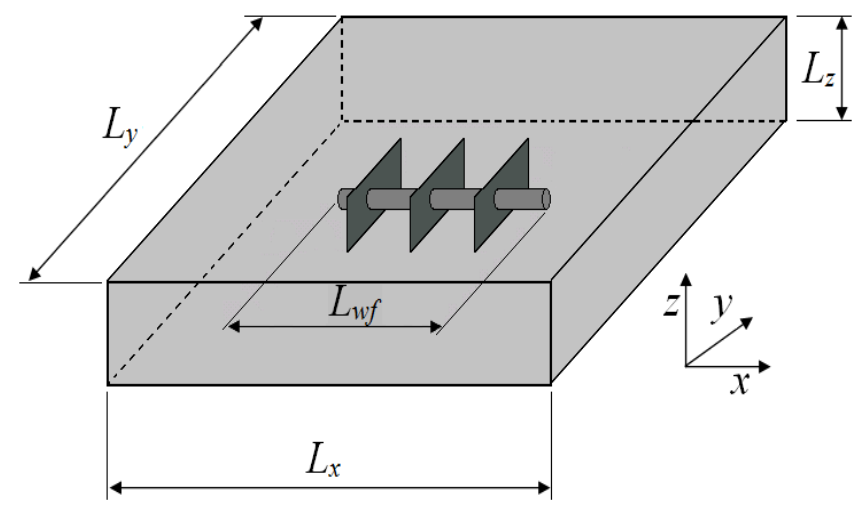

Figura 2: Ilustração de reservatório com poço de produção horizontal hidraulicamente fraturado.

Raghavan et al. [12] apresentaram procedimentos de predição, incluindo uma solução semi-analítica, para determinar o desempenho de longo prazo da produção para poços horizontais interceptados por fraturas transversais. Os autores também demonstraram que existe um 
grande potencial de melhoramento da produtividade do poço a partir da introdução do fraturamento hidráulico não uniforme do poço horizontal de produção, ou seja, algumas regiões não sofrem o fraturamento.

As principais diferenças entre a produtividade de reservatórios empregando o fraturamento hidráulico e poços horizontais e verticais foram estudadas por [13]. Os resultados mostraram que a produtividade pode ser aumentada em até cinco vezes quando um poço horizontal, longitudinalmente fraturado, é utilizado, ao invés de poços verticais hidraulicamente fraturados. As pesquisas consideraram os casos de produção a pressão e a vazão constantes.

No contexto de reservatórios de muito baixa permeabilidade, Bello e Wattenbarger [14] desenvolveram um modelo transiente para o fraturamento hidráulico multiestágio de poços para formações de gás do tipo shale gas. Cinco regimes de escoamento foram identificados pelos autores:

1. Linear inicial, da rede de fraturas para o poço;

2. Linear transiente, através das fraturas e da matriz sólida:

3. Linear tardio para um reservatório infinito, também descrito como escoamento transiente linear na matriz para grandes reservatórios;

4. Linear tardio para reservatórios fechados, também conhecido como escoamento linear transiente na matriz sólida;

5. Dominado pelos efeitos de fronteira.

A solução em tempo real foi fornecida para cada um dos cinco regimes de escoamento.

\section{2 Óleo leve}

Com o crescimento do consumo energético em todo o mundo, os reservatórios convencionais que são considerados reservatórios de "fácil produção" não estão atendendo à demanda energética mundial, fazendo-se necessário a explotação de reservas não convencionais, que são caracterizadas por suas baixas permeabilidade e porosidade ou alta viscosidade [11], vide a Fig. 3. Uma das aplicações que vem ganhando grande destaque é a utilização de poços horizontais hidraulicamente fraturados em reservatórios de baixa permeabilidade portadores de óleo leve.

O óleo ainda no reservatório é formado por uma mistura de hidrocarbonetos. Comumente os hidrocarbonetos de cadeia mais longas (com mais de 70 átomos de carbono) são chamados de óleo pesado e os de cadeia mais curta (com cerca de 10 átomos de carbono) de óleo leve. Essa diferença de composição química, basicamente, depende dos processos iniciais de sua formação e do local do reservatório. O conhecimento sobre qual tipo de óleo está

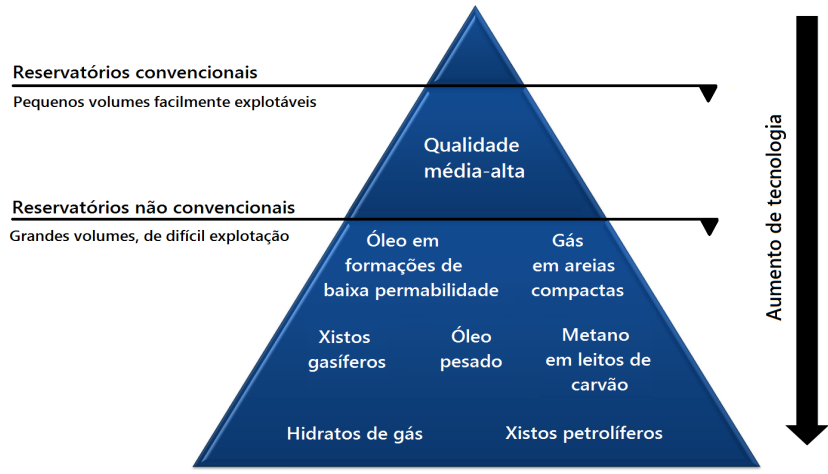

Figura 3: Ilustração de recursos convencionais e não convencionais.

no reservatório é fundamental para o desenvolvimento de um projeto de recuperação.

O óleo bruto é classificado, em função de sua densidade de hidrocarbonetos e da sua capacidade de fluir, como: leve, médio, pesado e extrapesado. A escala usada para indicar a massa específica do óleo é o Grau API (API Gravity, em inglês) criada pelo American Petroleum Institute, calculado como

$$
A P I=\left(\frac{141,5}{d_{60 / 60^{\circ} \mathrm{F}}}\right)-131,5,
$$

onde $d_{60 / 60^{\circ} \mathrm{F}}$ é a densidade do óleo a 60 graus Fahrenheit [7] com relação à água (na mesma temperatura). A escala API é medida em graus e permite definir o óleo como [15]:

1. Óleo leve: possui API maior que 30, constituído basicamente por alcanos, e uma porcentagem de 15 a $25 \%$ de cicloalcanos;

2. Óleo médio: possui API de 22 a 30 . Além de alcanos, contém de 25 a $30 \%$ de hidrocarbonetos aromáticos;

3. Óleo pesado: possui API menor que 22 e é composto só de hidrocarbonetos aromáticos;

4. Óleo extrapesado: possui API menor que 10, é constituído de hidrocarbonetos de cadeia longa (superior ao pentano).

O óleo leve, denominado Light Tight Oil (LTO), é o óleo contido em formações de baixa permeabilidade, muitas vezes, folhelhos ou arenitos. O recurso (um recurso mineral é uma concentração ou ocorrência de material de interesse econômico intrínseco na superfície ou no interior da crosta terrestre) de LTO corresponde a um quarto do tamanho dos oil sands (mistura que ocorre naturalmente de areia, argila ou outros minerais, água e betume) e a duas vezes os recursos globais em águas profundas.

O objetivo deste trabalho consiste no uso do nosso simulador numérico de reservatórios, para o estudo da influência do emprego de poços horizontais hidraulicamente fraturados na determinação do campo de pressão 
para o escoamento de um óleo leve, em formações de baixa permeabilidade, considerando os efeitos combinados das fraturas hidráulicas e do poço horizontal. Cabe salientar que a presença de não-linearidades contribui sobremaneira no comportamento das soluções obtidas e deve ser levada em consideração quando da escolha do método numérico a ser empregado. Por outro lado, a distribuição e o grau das heterogeneidades tem influência direta na determinação dos campos de pressão e de velocidade no meio poroso. O impacto das heterogeneidades também pode ser detectado no comportamento da pressão no poço de produção. Portanto, investiga-se os efeitos combinados das fraturas hidráulicas com o poço horizontal, considerando diferentes cenários de produção de óleo leve, no perfil da perda de pressão no poço produtor.

\section{Modelagem de escoamentos em meios porosos}

Em um reservatório, os fluidos presentes interagem entre si e com o reservatório portador de hidrocarbonetos. Esta interação depende das características dos fluidos envolvidos no processo, tais como a viscosidade e a massa específica e das características do meio, como a porosidade e a permeabilidade. Para se descrever o escoamento dos fluidos, ter o conhecimento dessas propriedades é essencial.

\subsection{Propriedades do fluido}

Revisa-se, agora, algumas propriedades de fluido incluídas na modelagem do escoamento monofásico em meios porosos. Primeiramente, considera-se a massa específica, que é a relação entre a massa de uma determinada substância e o volume ocupado por ela, que é definida para todo material como

$$
\rho=\frac{m}{V}
$$

onde $m$ representa a massa da substância e $V$ o volume por ela ocupado.

Uma outra propriedade relevante na modelagem do escoamento em meios porosos é a compressibilidade isotérmica do fluido,

$$
c_{o}=-\left[\frac{1}{V} \frac{\partial V}{\partial p}\right]_{T}=\left[\frac{1}{\rho} \frac{\partial \rho}{\partial p}\right]_{T} .
$$

sendo que $p$ representa a pressão e $T$ a temperatura.

A partir da compressibilidade do óleo, a seguinte equação para cálculo de $\rho$ pode ser obtida [16]

$$
\rho=\rho^{0}\left[1+c_{o}\left(p-p^{0}\right)\right]
$$

onde $\rho^{0}$ é a massa específica medida na pressão de referência $p^{0}$.

A medida do quão facilmente o fluido se deforma quando submetido à aplicação de um gradiente de pressão é denominada viscosidade, $\mu$. A viscosidade de um fluido é função da pressão e da temperatura,

$$
\mu=\mu(p, T)
$$

Uma das formas utilizadas para o cálculo da viscosidade no caso de um fluido ligeiramente compressível é

$$
\mu=\frac{\mu^{0}}{\left[1+c_{\mu}\left(p-p^{0}\right)\right]}
$$

onde $\mu^{0}$ é a viscosidade medida na pressão de referência $p^{0}$ e $c_{\mu}$ é o coeficiente que mede a taxa de variação da viscosidade com a pressão.

Finalmente, introduz-se o fator-volume-formação, $B$,

$$
B=\frac{V(p, T)}{V_{s c}}=\frac{\rho_{s c}}{\rho},
$$

onde o subscrito $s c$ indica as condições padrão e $p$ e $T$ são a pressão e a temperatura, respectivamente [17]. Considerando $B^{0}$ o fator-volume-formação medido na pressão de referência $p^{0}$, para um fluido ligeiramente compressível é possível utilizar a relação [16]

$$
B=\frac{B^{0}}{\left[1+c_{o}\left(p-p^{0}\right)\right]} .
$$

\subsection{Propriedades da rocha}

Inicia-se pela porosidade, a qual mede a capacidade de armazenamento de fluidos no reservatório. Ela é definida como sendo dada pela relação:

$$
\phi=\frac{V_{p}}{V_{t}},
$$

onde $\phi$ é a porosidade, $V_{t}$ o volume total e $V_{p}$ o volume de vazios que também pode ser denominado de volume poroso [17].

Em uma rocha reservatório podem ser encontrados dois tipos de porosidade, as porosidades efetiva e total. A porosidade efetiva contempla apenas os poros interconectados [19]. Por outro lado, a porosidade total inclui os poros isolados e os interconectados. Para a Engenharia de Reservatórios, a porosidade efetiva é de maior interesse pois apenas poros interconectados permitem a percolação de fluidos através da rocha. A porosidade geralmente varia no espaço ao longo de uma formação rochosa e o reservatório é dito ser heterogêneo.

Vale destacar que, contudo, uma porosidade alta não é suficiente para caracterizar a potencialidade econômica de um reservatório, pois os fluidos do reservatório de petróleo contidos nos espaços dos poros das rochas tem que fluir para que então sejam produzidos ou trazidos para a superfície. Isto depende de uma propriedade da rocha, denotada por $k$, chamada de permeabilidade [18].

A permeabilidade pode variar em um mesmo ponto, em função da direção, e ao longo do meio poroso. Em muitas aplicações práticas é razoável considerar que a permeabilidade pode ser representada somente nas três direções principais em coordenadas cartesianas, $x, y$ e $z$ por $k_{x}, k_{y}$ e $k_{z}$, respectivamente. O meio poroso é classificado como isotrópico se $k_{x}=k_{y}=k_{z}$ e se a permeabilidade apresenta variação segundo as direções espaciais é denominado de anisotrópico. 
A compressibilidade da rocha é definida como sendo

$$
c_{\phi}=\left[\frac{1}{\phi} \frac{\partial \phi}{\partial p}\right]
$$

A partir da compressibilidade da rocha pode-se escrever [16]

$$
\phi=\phi^{0}\left[1+c_{\phi}\left(p-p^{0}\right)\right]
$$

onde $\phi^{0}$ é a porosidade medida na pressão de referência $p^{0}$.

\subsection{Equações fundamentais}

As hipóteses utilizadas na derivação do modelo físicomatemático que descreve o escoamento no reservatório são:

1. O meio poroso é heterogêneo e anisotrópico;

2. A compressibilidade da rocha é pequena e constante;

3. O fluido é newtoniano;

4. Não ocorrem reações químicas;

5. O escoamento ocorre a baixas velocidades (efeitos inerciais não considerados);

6. O fluido tem uma composição constante;

7. O escoamento é monofásico e isotérmico;

8. Poço horizontal com condutividade infinita; e

9. Ausência de estocagem no poço.

\subsubsection{Lei de Darcy}

Tipicamente, a equação usada para descrever a conservação da quantidade de movimento no escoamento de fluidos em meios porosos é a lei de Darcy,

$$
\mathbf{v}=-\frac{\mathbf{k}}{\mu}[\nabla p-\rho g \nabla D],
$$

onde $\mathbf{v}$ é a velocidade superficial do fluido, $\mathbf{k}$ é o tensor de permeabilidades, $D$ é a profundidade e $g$ é a magnitude da aceleração da gravidade [17].

\subsubsection{Equação de continuidade}

A equação de conservação da massa é escrita em termos do fator-volume-formação $B$ [17]

$$
\frac{\partial}{\partial t}\left(\frac{\phi}{B}\right)+\nabla \cdot\left(\frac{\mathbf{v}}{B}\right)-\frac{q_{m}}{V_{b} \rho_{s c}}=0
$$

onde $q_{m}$ é o termo fonte, $V_{b}$ é o volume total do volume de controle (rocha mais poros) e $\rho_{s c}$ representa a massa específica em condições padrão.

\subsubsection{Introdução das compressibilidades}

Utilizando-se a regra da cadeia pode-se expandir o termo $\partial(\phi / B) / \partial t[16]$

$$
\begin{aligned}
\frac{\partial}{\partial t}\left(\frac{\phi}{B}\right) & =\frac{1}{B} \frac{\partial \phi}{\partial p} \frac{\partial p}{\partial t}+\phi \frac{\partial}{\partial p}\left(\frac{1}{B}\right) \frac{\partial p}{\partial t} \\
& =\left[\frac{1}{B} \frac{\partial \phi}{\partial p}+\phi \frac{\partial}{\partial p}\left(\frac{1}{B}\right)\right] \frac{\partial p}{\partial t} .
\end{aligned}
$$

e, usando-se as Eqs. (8) e (11),

$$
\frac{\partial}{\partial t}\left(\frac{\phi}{B}\right)=\left(\frac{\phi c_{o}}{B^{0}}+\frac{\phi^{0} c_{\phi}}{B}\right) \frac{\partial p}{\partial t} .
$$

\subsection{Equação da difusividade hidráulica}

Substituindo-se a Eq. (7) e a Eq. (12) na Eq. (13), multiplicando-se por $V_{b}$, introduzindo-se $q_{m}=q_{s c} \rho_{s c}$ e utilizando-se a Eq. (15) para reescrever-se a derivada temporal, obtém-se uma equação diferencial parcial nãolinear:

$$
\Gamma \frac{\partial p}{\partial t}-V_{b} \nabla \cdot\left(\frac{\mathbf{k}}{B \mu} \nabla p\right)+\Gamma_{G}-q_{s c}=0,
$$

onde [16]

$$
\Gamma=V_{b}\left(\frac{\phi c_{o}}{B^{0}}+\frac{\phi^{0} c_{\phi}}{B}\right),
$$

sendo que $\Gamma_{G}$ inclui os efeitos gravitacionais.

O termo fonte $q_{s c}$ é usado para introduzir-se a pressão do poço, $p_{w f}$, através da relação

$$
q_{s c}=-J_{w}\left(p-p_{w f}\right),
$$

onde $J_{w}$ é o Índice de Produtividade (IP) [20].

Para resolver-se a Eq. (16) são necessárias condições inicial e de contorno apropriadas. Como condição inicial utiliza-se

$$
p(x, y, z, t=0)=p_{\text {ini }}(x, y, z),
$$

onde a pressão inicial antes do reservatório ser perturbado pela produção/injeção é representada por $p_{\text {ini } i}$.

As condições de contorno externas são as de fluxo nulo nas fronteiras

$$
\left(\frac{\partial p}{\partial x}\right)_{x=0, L_{x}}=\left(\frac{\partial p}{\partial y}\right)_{y=0, L_{y}}=\left(\frac{\partial p}{\partial z}\right)_{z=0, L_{z}}=0
$$

onde $L_{x}, L_{y}$ e $L_{z}$ são os respectivos comprimentos do reservatório nas direções $x, y$ e $z$.

A condição de contorno interna é de vazão de produção prescrita. Para o acoplamento poço-reservatório linearizado, para um fluxo de fase única, é necessário uma equação específica para a determinação do fluxo, de modo a calcular-se o índice $J_{w}$ e a pressão no poço será uma das incógnitas a serem determinadas. Neste trabalho, considera-se um poço horizontal, desprezando-se o atrito e os efeitos inerciais do escoamento dentro do poço. O fluxo total do poço, $Q_{s c}$, deve ser igual ao fluxo deixando o reservatório e atravessando toda a superfície do poço de produção em contato com o reservatório $\left(q_{s c}\right)$. 


\subsection{Regimes de escoamento para um poço horizontal fraturado}

Na caracterização dos regimes de escoamento, a utilização da derivada da pressão em função do tempo é matematicamente adequada, porque essa derivada representa diretamente um termo da equação de difusividade, que é a equação utilizada para a modelagem do comportamento da pressão transiente na análise de testes de pressão de poços [1]. A derivada é mais sensível a pequenas variações em fenômenos de interesse como no caso da variação da pressão em função do tempo [21].

Uma ferramenta importante na análise de testes de pressão é a utilização do gráfico diagnóstico (log-log). Nesse gráfico são apresentados a variação da pressão no poço e da sua derivada em função do tempo transcorrido, ou seja, a derivada de Bourdet [22]:

$$
\Delta p=\frac{d p}{d \ln \Delta t}=\Delta t \frac{d p}{d t} .
$$

Para melhor visualização dos resultados obtidos, também foi utilizado o gráfico especializado, ou seja, pressão no poço em função do logaritmo do tempo. O estudo da queda de pressão no poço e da sua derivada têm sido de grande importância na análise de testes de pressão. A introdução dos métodos das derivadas proporcionou um grande avanço na identificação dos regimes de escoamento que podem ocorrer para cada tipo de reservatório e melhorou significativamente o diagnóstico, de forma que estes gráficos são importantes ferramentas também para a simulação de reservatórios [23].

Com base nas características do óleo encontrado em formação de baixa permeabilidade, tight oil, e métodos de produção de reservatório, existem quatro configurações [24]:

1. Presença de poço horizontal;

2. Presença de poço horizontal hidraulicamente fraturado;

3. Estimulação de volume, em um único estágio, ao redor do poço horizontal hidraulicamente fraturado e

4. Estimulação de volume, em multi-estágios, ao redor do poço horizontal hidraulicamente fraturado.

Investiga-se, aqui, o escoamento em um reservatório com um poço horizontal hidraulicamente fraturado, o qual pode ser dividido em seis regimes de escoamento [24]:

\section{Estocagem no poço;}

2. Linear da fratura, no qual o escoamento do fluido é perpendicular à superfície da fratura;

3. Pseudo-radial da fratura. Quanto mais curto a metade do comprimento da fratura mais torna-se visível o escoamento pseudo-radial, para o mesmo espaçamento da fratura;
4. Linear do sistema; a interferência da fratura aparece e, em seguida, o fluido flui principalmente da zona exterior à zona interior do sistema do poço horizontal e das fraturas;

5. O sistema apresenta um escoamento radial; a pressão no sistema de multi-fraturas é quase como um todo destinada para possibilitar o fluxo pseudoradial; e

6. Dominância dos efeitos da fronteira externa do reservatório.

\section{Solução Numérica}

A solução das equações que descrevem o escoamento de um fluido em meios porosos é fundamental para a compreensão do fenômeno estudado. Estas equações, conforme visto, são obtidas usando a hipótese de meio contínuo e os balanços de massa e da quantidade de movimento. No entanto, como no caso geral não é possível obter-se uma solução analítica, utiliza-se uma abordagem numérica, obtendo-se os valores da variável dependente (a pressão) em pontos discretos do domínio de resolução.

\subsection{Discretização da equação de difusividade} hidráulica

O método de discretização empregado neste trabalho é o método de diferenças finitas, usado no caso de uma malha de blocos centrados [16]. Uma representação esquemática de um domínio tridimensional discretizado pode ser vista na Fig. 4 para o sistema de coordenadas cartesianas. A solução numérica é obtida nos nós da malha computacional e o sistema algébrico de equações resultante do processo de discretização da EDP é resolvido por um método iterativo, que no caso deste trabalho será o método dos Gradientes Conjugados Pré-condicionado.

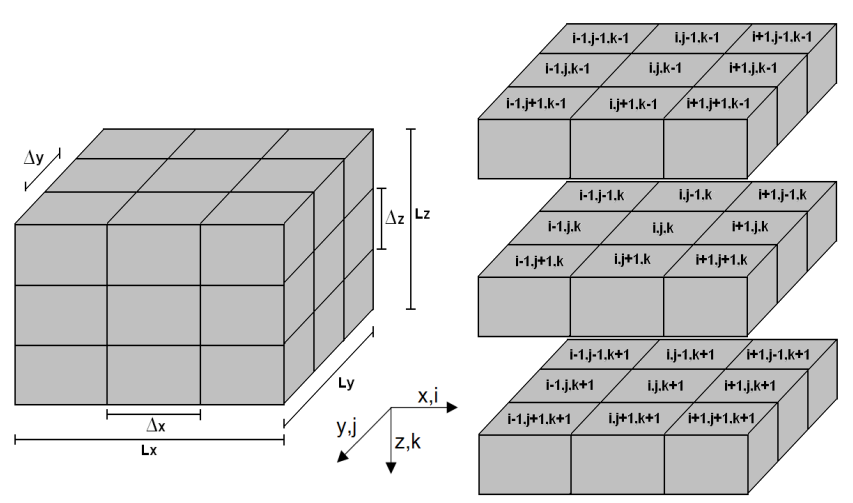

Figura 4: Representação de um domínio tridimensional discretizado. 
Substituindo-se a Eq. (18) na Eq. (16), para um problema tridimensional, pode-se obter a Equação de Difusividade Hidráulica na forma

$$
\begin{gathered}
\quad \frac{\partial}{\partial x}\left[\left(\frac{A_{x} k_{x}}{\mu B}\right) \frac{\partial p}{\partial x}\right] \Delta x+\frac{\partial}{\partial y}\left[\left(\frac{A_{y} k_{y}}{\mu B}\right) \frac{\partial p}{\partial y}\right] \Delta y \\
+\frac{\partial}{\partial z}\left[\left(\frac{A_{z} k_{z}}{\mu B}\right) \frac{\partial p}{\partial z}\right] \Delta z=\Gamma \frac{\partial p}{\partial t}+\Gamma_{G}+J_{w}\left(p-p_{w f}\right),
\end{gathered}
$$

onde $V_{b}=\Delta x \Delta y \Delta z, A_{x}=\Delta y \Delta z, A_{y}=\Delta x \Delta z$ e $A_{z}=$ $\Delta x \Delta y$.

Introduzindo-se as novas variáveis

$$
T_{x}^{\prime} \equiv \frac{A_{x} k_{x}}{\mu B}, \quad T_{y}^{\prime} \equiv \frac{A_{y} k_{y}}{\mu B} \quad \text { e } \quad T_{z}^{\prime} \equiv \frac{A_{z} k_{z}}{\mu B},(23)
$$

a Eq. (22) pode ser reescrita como

$$
\begin{aligned}
& \frac{\partial}{\partial x}\left(T_{x}^{\prime} \frac{\partial p}{\partial x}\right) \Delta x+\frac{\partial}{\partial y}\left(T_{y}^{\prime} \frac{\partial p}{\partial y}\right) \Delta y+\frac{\partial}{\partial z}\left(T_{z}^{\prime} \frac{\partial p}{\partial z}\right) \Delta z \\
= & \Gamma \frac{\partial p}{\partial t}+\Gamma_{G}+J_{w}\left(p-p_{w f}\right) .
\end{aligned}
$$

Considerando-se um arranjo de malha computacional como o apresentado na Fig. 4 obtém-se [16]

$$
\begin{aligned}
\frac{\partial}{\partial x}\left(T_{x}^{\prime} \frac{\partial p}{\partial x}\right)_{i, j, k}^{n+1} & \cong \frac{1}{\Delta x_{i, j, k}}\left[\left(T_{x}^{\prime} \frac{\partial p}{\partial x}\right)_{i+\frac{1}{2}, j, k}\right. \\
& \left.-\left(T_{x}^{\prime} \frac{\partial p}{\partial x}\right)_{i-\frac{1}{2}, j, k}\right]^{n+1},
\end{aligned}
$$

onde $\Delta x_{i, j, k}$ é o espaçamento da malha na direção $x$, $n+1$ representa o nível temporal em que as pressões são calculadas ( $n$ indica o nível de tempo em que a pressão é conhecida). Em seguida, a fim de aproximar-se as derivadas espaciais, considera-se inicialmente o problema da discretização na direção $x$ do espaço. Os nós $i-1 / 2$ e $i+1 / 2$ representam as faces laterais da célula e o nó $i$ o seu centro, de forma que

$$
\begin{aligned}
& \left(\frac{\partial p}{\partial x}\right)_{i+\frac{1}{2}, j, k}^{n+1} \cong \frac{p_{i+1, j, k}^{n+1}-p_{i, j, k}^{n+1}}{\Delta x_{i+\frac{1}{2}, j, k}} \\
& \left(\frac{\partial p}{\partial x}\right)_{i-\frac{1}{2}, j, k}^{n+1} \cong \frac{p_{i, j, k}^{n+1}-p_{i-1, j, k}^{n+1}}{\Delta x_{i-\frac{1}{2}, j, k}}
\end{aligned}
$$

onde $\Delta x_{i \pm 1 / 2, j, k}$ é a distância entre os centros das células representadas por $i$ e $i \pm 1$. Analogamente, é possível obter-se aproximações para as derivadas nas direções $y$ $\mathrm{e} z$.

A transmissibilidade na direção $x$ pode ser introduzida como

$$
T_{x, i \pm \frac{1}{2}, j, k}^{n+1}=\left(\frac{A_{x} k_{x}}{\mu B \Delta x}\right)_{i \pm \frac{1}{2}, j, k}^{n+1},
$$

onde é utilizada uma média harmônica para determinarse a transmissibilidade em $i \pm 1 / 2, j, k$ a partir dos valores conhecidos em $i, j, k$ e $i \pm 1, j, k$. Similarmente, expressões podem ser determinadas para as transmissibilidades nas direções $y$ e $z$.
Finalmente, utilizando-se uma formulação totalmente implícita no tempo, é possível obter-se a forma final discretizada da Eq. (22)

$$
\begin{aligned}
& T_{x, i+\frac{1}{2}, j, k}^{n+1}\left(p_{i+1, j, k}^{n+1}-p_{i, j, k}^{n+1}\right)-T_{x, i-\frac{1}{2}, j, k}^{n+1}\left(p_{i, j, k}^{n+1}-p_{i-1, j, k}^{n+1}\right) \\
+ & T_{y, i, j+\frac{1}{2}, k}^{n+1}\left(p_{i, j+1, k}^{n+1}-p_{i, j, k}^{n+1}\right)-T_{y, i, j-\frac{1}{2}, k}^{n+1}\left(p_{i, j, k}^{n+1}-p_{i, j-1, k}^{n+1}\right) \\
+ & T_{z, i, j, k+\frac{1}{2}}^{n+1}\left(p_{i, j, k+1}^{n+1}-p_{i, j, k}^{n+1}\right)-T_{z, i, j, k-\frac{1}{2}}^{n+1}\left(p_{i, j, k}^{n+1}-p_{i, j, k-1}^{n+1}\right) \\
= & \Gamma_{i, j, k}^{n+1}\left(p_{i, j, k}^{n+1}-p_{i, j, k}^{n}\right)+\left(\Gamma_{G}\right)_{i, j, k}^{n+1} \\
+ & J_{w, i, j, k}^{n+1}\left(p_{i, j, k}^{n+1}-p_{w f, i, j, k}^{n+1}\right) .
\end{aligned}
$$

onde foi utilizada uma aproximação de Euler recuada no tempo

$$
\left(\frac{\partial p}{\partial t}\right)_{i, j, k}^{n+1} \cong \frac{p_{i, j, k}^{n+1}-p_{i, j, k}^{n}}{\Delta t}
$$

\subsection{Refinamento de malha}

Um refinamento de malha é usado para uma melhor representação da região ao redor do poço horizontal. Por exemplo, para um poço perpendicular ao plano $y z$, o espaçamento da malha na direção $y, \Delta y_{i, j, k}$, e na direção $z, \Delta z_{i, j, k}$, são menores perto das células onde está presente o poço horizontal. Considerando-se os índices $i, j$ e $k$, conforme apresentado na Fig 4, utilizados na discretização, a Fig. 5 exibe uma representação do refinamento de malha em torno da localização do poço horizontal. $\Delta y_{w}$ e $\Delta z_{w}$ são escolhidos para as células onde o poço é colocado. Para as outras células, $\Delta y_{i, j, k}$ e $\Delta z_{i, j, k}$ são pequenos perto do poço, e eles aumentam a uma determinada taxa, conforme as células estejam mais distantes do poço horizontal.

Uma progressão logarítmica é utilizada no refinamento da malha. Por exemplo, para uma fratura colocada no plano $y z$, as larguras da malha na direção $x$, $\Delta x_{i, j, k}$, são menores perto das células utilizadas para modelar a fratura, como representado na Fig. 6. Dentro de uma célula original, um conjunto de pequenos blocos é adicionado a fim de representar a fratura e criar uma região de transição.

Tomando-se como exemplo uma fratura colocada no plano $y z$, uma constante $\Delta U_{x}$ é definida como [25]

$$
\Delta U_{x}=\frac{2}{\left(n_{C X}-1\right)} \ln \left(\frac{x_{\max }}{x_{\min }}\right),
$$

e o espaçamento das células ao longo da direção $x$, na região de transição, é determinado por

$$
\Delta x_{I \pm i, j, k}=x_{i n i}\left[\mathrm{e}^{i \Delta U_{x}}-\mathrm{e}^{(i-1) \Delta U_{x}}\right]
$$

para $i=1,2, \ldots,\left(n_{C X}-1\right) / 2$, onde $I$ indica um plano $y z$ em que há uma fratura, $\Delta x_{I, j, k}$ é a espessura da fratura, $n_{C X}$ é o número (ímpar) de camadas refinadas na direção $x$ e $x_{\min }$ e $x_{\max }$ são as posições inicial e final da região de transição, respectivamente. Cada fratura é rodeada por duas regiões refinadas ao longo da direção 
$x$ e $x_{\text {min }}$ é escolhido como sendo metade da espessura da fratura, $h_{f}$, enquanto $x_{\max }$ é a metade da largura da célula original na direção $x$.
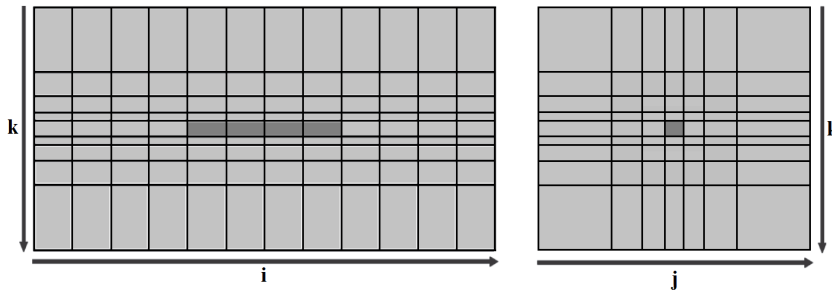

Figura 5: Malha refinada para representação de poço horizontal perpendicular ao plano $y z$. À esquerda o plano $x z$; ̀̀ direita o plano $y z$; e, em cor cinza escuro, as células pelas quais passa o poço horizontal.

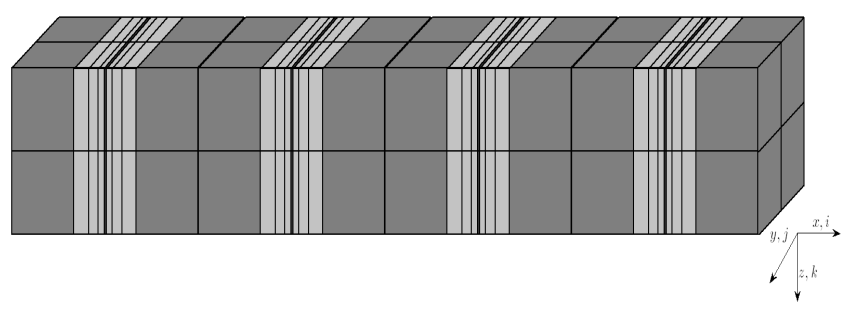

Figura 6: Ilustração do refinamento de malha para melhor representação do escoamento na região das fraturas.

\subsection{Linearização do sistema de equações algébricas não-lineares}

Escrito para cada célula, a Eq. (29) conduz a um conjunto de equações algébricas não-lineares em termos da incógnita pressão. Para utilizar-se técnicas de solução de sistemas de equações lineares, tem-se que linearizar as equações algébricas. Considerando-se a aplicação de uma iteração de Picard, para os termos da transmissibilidade [26], introduz-se a aproximação

$$
T_{x, i \pm \frac{1}{2}, j, k}^{n+1} \cong T_{x, i \pm \frac{1}{2}, j, k}^{n+1, v}=\left(\frac{A_{x} k}{\mu B \Delta x}\right)_{i \pm \frac{1}{2}, j, k}^{n+1, v},
$$

de modo que a Eq. (29) pode ser reescrita como

$$
\begin{aligned}
& T_{x, i+\frac{1}{2}, j, k}^{n+1, v}\left(p_{i+1, j, k}^{n+1, v+1}-p_{i, j, k}^{n+1, v+1}\right) \\
- & T_{x, i-\frac{1}{2}, j, k}^{n+1, v}\left(p_{i, j, k}^{n+1, v+1}-p_{i-1, j, k}^{n+1, v+1}\right) \\
+ & T_{y, i, j+\frac{1}{2}, k}^{n+1, v}\left(p_{i, j+1, k}^{n+1, v+1}-p_{i, j, k}^{n+1, v+1}\right) \\
- & T_{y, i, j-\frac{1}{2}, k}^{n+1, v}\left(p_{i, j, k}^{n+1, v+1}-p_{i, j-1, k}^{n+1, v+1}\right) \\
+ & T_{z, i, j, k+\frac{1}{2}}^{n+1, v}\left(p_{i, j, k+1}^{n+1, v+1}-p_{i, j, k}^{n+1, v+1}\right) \\
- & T_{z, i, j, k-\frac{1}{2}}^{n+1, v}\left(p_{i, j, k}^{n+1, v+1}-p_{i, j, k-1}^{n+1, v+1}\right) \\
= & \Gamma_{i, j, k}^{n+1, v}\left(p_{i, j, k}^{n+1, v+1}-p_{i, j, k}^{n}\right)+\left(\Gamma_{G}\right)_{i, j, k}^{n+1} \\
+ & J_{w, i, j, k}^{n+1, v}\left(p_{i, j, k}^{n+1, v+1}-p_{w f, i, j, k}^{n+1, v+1}\right)
\end{aligned}
$$

onde os níveis de iteração são indicados por $v$ (valores conhecidos) e $v+1$ (valores desconhecidos). O mesmo procedimento é utilizado para o termos $\Gamma_{i, j, k}$ e $J_{w}$.

Para descrever-se o acoplamento poço-reservatório também lineariza-se o termo $J_{w}$. Aqui, apenas poços horizontais são considerados, negligenciando-se as perdas de carga e os efeitos inerciais dentro do poço. A taxa de fluxo total do poço, $Q_{s c}$, deve ser igual à soma do fluxo de óleo oriundo de todas as células em contato com o poço, portanto,

$$
Q_{s c}=-\sum_{i=W 1}^{i=W 2} J_{w, i, j, k}^{n+1, v}\left[p_{i, j, k}^{n+1, v+1}-\left(p_{w f}\right)_{i, j, k}^{n+1, v+1}\right] .
$$

para um poço atravessando várias camadas ao longo da direção $x$, para células representadas por $W 1, j, k$ (inicial) a $W 2, j, k$ (final). A posição de referência, em $W 1, j, k$, é escolhida a fim de calcular-se a pressão do poço.

Para $J_{w}$ utiliza-se [27]

$$
J_{w, i, j, k}^{n+1, v}=\left\{\frac{2 \pi \sqrt{k_{z} k_{y}}}{\mu B} \frac{\Delta x}{\left[1-\left(r_{w} / r_{e q}\right)^{2}\right] \ln \left(r_{e q} / r_{w}\right)}\right\}_{i, j, k}
$$

onde

$$
r_{e q}=\sqrt{\frac{\Delta z \Delta y}{\pi}} \exp (-0,5)
$$

com $r_{e q}$ sendo o raio equivalente [28] e $r_{w}$ é o raio do poço.

\subsection{Metodologia de resolução do sistema linear}

O sistema linear oriundo da linearização das equações algébricas não-lineares tem em geral, como característica, a esparsidade da matriz de coeficientes e a numerosa quantidade de incógnitas. Devido ao porte do sistema, é necessária uma grande quantidade de memória e velocidade de processamento para a sua resolução. O método dos Gradientes Conjugados Pré-condicionado, com um pré-condicionador de Jacobi [16], foi empregado com essa finalidade.

\section{Resultados}

Esta seção é dedicada à apresentação dos resultados e dos comentários acerca das simulações realizadas, em conformidade com a estratégia de solução numérica já discutida e empregando-se o simulador previamente introduzido. Em todos os casos testados considera-se que o poço produtor horizontal está posicionado paralelamente ao eixo $x$. O poço horizontal recebe fluido vindo do reservatório em todo o seu comprimento, em uma completação de poço aberto.

O simulador utilizado para realizar este trabalho foi escrito em linguagem $\mathrm{C}$ e as simulações foram feitas em um computador DELL PowerEdge T620 com sistema operacional Linux OpenSuse 13.1: 
1. Máquina de arquitetura 64 bits;

2. Processador Intel Xeon E5-2620 com 2 GHz e 12 núcleos.

\subsection{Simulação de poço horizontal}

A Tabela 1 traz os parâmetros utilizados na construção de um caso padrão de simulação. A menos quando explicitamente dito, os dados apresentados nessa tabela são os usados em todas as simulações deste trabalho.

Fazendo-se uma simulação numérica com esses parâmetros são determinados os campos espaciais de pressão. O refinamento local da malha foi utilizado no estudo de poços horizontais fraturados hidraulicamente, em conjunto com a técnica de acoplamento poço-reservatório proposta neste trabalho. Uma regra logarítmica foi considerada para a geração da malha refinada, nas regiões contendo as fraturas, considerando-se o poço posicionado paralelamente ao eixo $x$

A Tabela 2 mostra os números de células empregadas na geração de quatro diferentes malhas para o estudo de convergência numérica, considerando as simulações envolvendo o poço horizontal sem as fraturas hidráulicas, onde $n_{x}, n_{y}$ e $n_{z}$ são, respectivamente, os números de células usadas para representar o reservatório nas direções $x, y$ e $z$. O termo $n_{w}$ representa o número de células na direção $x$ pelas quais o poço horizontal passa no reservatório. Também em relação às células por onde passa o poço, os valores $\Delta y_{w}$ e $\Delta z_{w}$ são as arestas das células que contém o poço, respectivamente, nas direções $y$ e $z$.

Tabela 2 Malhas para o estudo de refinamento em poço horizontal.

\begin{tabular}{lccccc}
\hline Malha & $n_{x}$ & $n_{y}$ & $n_{z}$ & $n_{w}$ & $\Delta y_{w}=\Delta z_{w}(f t)$ \\
\hline 1 & 12 & 13 & 5 & 4 & 50 \\
2 & 24 & 25 & 9 & 8 & 25 \\
3 & 48 & 49 & 17 & 16 & 12,5 \\
4 & 96 & 97 & 33 & 32 & 6,25 \\
\hline
\end{tabular}

As Figs. 7 e 8 mostram os resultados para o estudo de refinamento de malha onde os resultados obtidos foram para uma célula. Observa-se que conforme a malha é refinada as soluções numéricas tendem a ficar cada vez mais próximas umas das outras. A exceção ocorre em um trecho inicial da simulação, no qual ocorre um efeito conhecido na literatura como "artefato numérico", que está associado à técnica de acoplamento poço-reservatório utilizada, baseada na hipótese do regime permanente. Também é possível notar que para as duas malhas mais refinadas há uma significativa concordância dos resultados, com exceção da região de influência do artefato numérico [27], destacando-se que o efeito do artefato é mais significativo somente para tempos muito curtos.

A pressão no poço horizontal foi calculada seguindo uma técnica do tipo raio equivalente de Peaceman [28]. Os resultados obtidos mostram que o artefato numérico influencia nos resultados obtidos para a pressão, nos tempos iniciais de simulação, e eles são caracterizados por

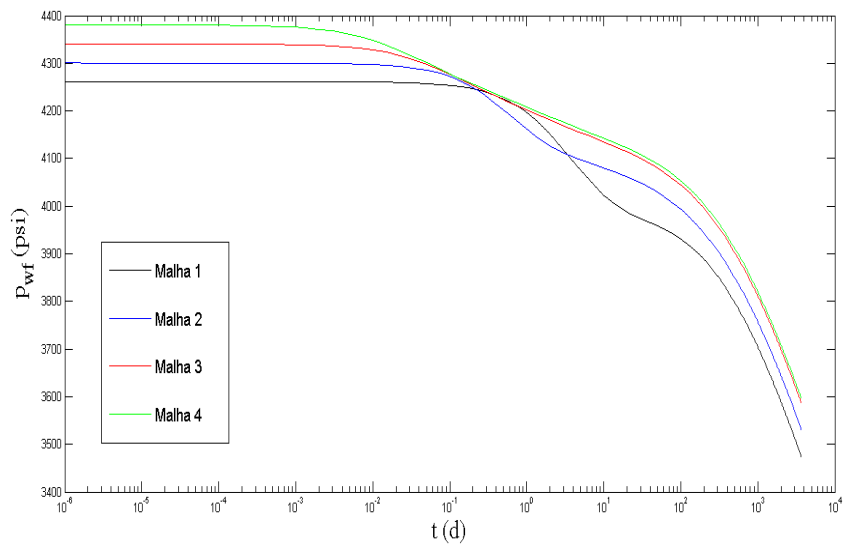

Figura 7: Resultado do refinamento de malha (gráfico especializado).

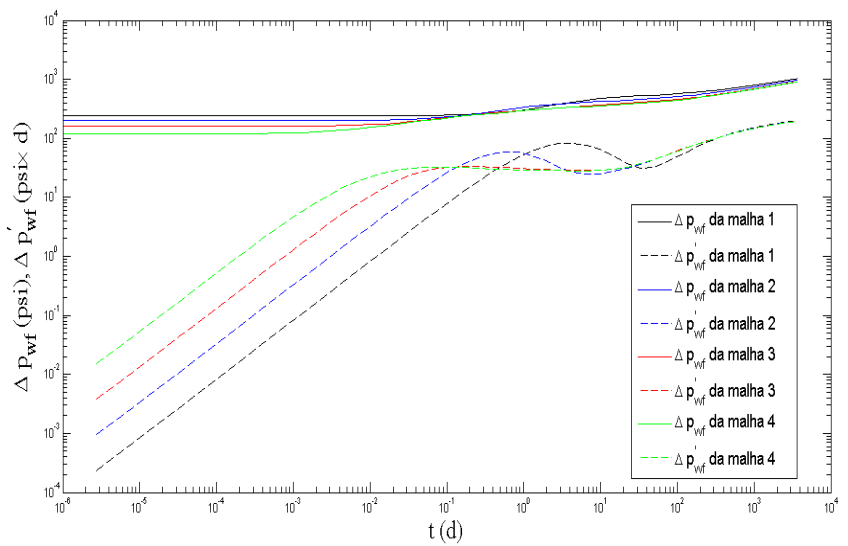

Figura 8: Resultado do refinamento de malha (gráfico diagnóstico).

um patamar no gráfico especializado e com reflexos na derivada presente nos gráficos diagnósticos. O tempo de ocorrência do artefato e a sua magnitude são funções da permeabilidade, sendo que quanto menor for a permeabilidade (e também para uma maior viscosidade) maior será sua duração e magnitude. Fora da região associada ao artefato, os comportamentos estão qualitativamente de acordo com a física associada ao problema. Então, neste trabalho, a fim de tratar-se especificamente do objetivo proposto, as análises irão se restringir às regiões de $10^{-2}$ dia em diante, em função dessa incorreção numérica [28].

\subsection{Simulação de poço horizontal hidraulicamente fraturado}

O caso padrão estudado, no que diz respeito à malha computacional utilizada, foi o correspondente à malha 3 , ou seja, 48 células na direção $x$, com a inclusão de 4 fraturas hidráulicas, distribuídas conforme mostrado na Fig. 9. Na determinação da influência das fraturas, na pressão do poço produtor, foram realizados testes com um poço horizontal com e sem fratura, Figs. 10, 11, 12 e 13. Por outro lado, a importância do número de fraturas $\left(n_{f}\right)$ e da posição espacial das fraturas $\left(p_{i}, i=\right.$ 
Tabela 1 Parâmetros para o caso padrão.

\begin{tabular}{lcc}
\hline Parâmetro & Valor & Unidade \\
\hline Número de células na direção $x$ & 48 & - \\
Número de células na direção $y$ & 49 & - \\
Número de células na direção $z$ & 17 & - \\
Tolerância: método iterativo & $1 \times 10^{-} 7$ & $\mathrm{psi}$ \\
Razão de crescimento do passo de tempo & 1,5 & - \\
Tempo de simulação & 3.650 & $\mathrm{dia}$ \\
Passo de tempo inicial & $1 \times 10^{-} 6$ & $\mathrm{dia}$ \\
Passo de tempo final & 30 & $\mathrm{dia}$ \\
Vazão de óleo & -200 & $\mathrm{STB} / \mathrm{dia}$ \\
Permeabilidade em $x$ & $2 \times 10^{-} 4$ & $\mathrm{Darcy}$ \\
Permeabilidade em $y$ & $2 \times 10^{-} 4$ & $\mathrm{Darcy}$ \\
Permeabilidade em $z$ & $5 \times 10^{-} 5$ & $\mathrm{Darcy}$ \\
Porosidade inicial & 0,1 & $\mathrm{adimensional}$ \\
Porosidade de referência & 0,1 & $\mathrm{adimensional}$ \\
Compressibilidade da rocha & $1 \mathrm{x} 10^{-} 5$ & $\mathrm{psi}$ \\
Pressão de referência & 4.500 & $\mathrm{psi}$ \\
Pressão inicial do fluido & 4.500 & $\mathrm{psi}$ \\
Massa específica do fluido na pressão de referência & 52,4 & $\mathrm{~kg} / \mathrm{m}^{3}$ \\
Viscosidade do fluido & 0,8 & $\mathrm{cp}$ \\
Compressibilidade do fluido & $4 \times 10^{-} 6$ & $\mathrm{psi}$ \\
Fator-volume-formação na pressão de referência & 1 & $\mathrm{RB} / \mathrm{STB}$ \\
Comprimento total na direção $x$ & 12.000 & $\mathrm{ft}$ \\
Comprimento total na direção $y$ & 12.000 & $\mathrm{ft}$ \\
Comprimento total na direção $z$ & 250 & $\mathrm{ft}$ \\
Comprimento do poço & 4.000 & $\mathrm{ft}$ \\
\hline
\end{tabular}

Tabela 3 Número de fraturas e suas respectivas posições.

\begin{tabular}{lccccccc}
\hline Caso & $n_{f}$ & $p_{1}$ & $p_{2}$ & $p_{3}$ & $p_{4}$ & $p_{5}$ & $p_{6}$ \\
\hline Caso 1 & 2 & $1.625 \mathrm{ft}$ & $2.375 \mathrm{ft}$ & - & - & - & - \\
Caso 2 & 4 & $1.125 \mathrm{ft}$ & $1.625 \mathrm{ft}$ & $2.375 \mathrm{ft}$ & $2.875 \mathrm{ft}$ & - & - \\
Caso 3 & 6 & $625 \mathrm{ft}$ & $1.125 \mathrm{ft}$ & $1.625 \mathrm{ft}$ & $2.375 \mathrm{ft}$ & $2.875 \mathrm{ft}$ & $3.375 \mathrm{ft}$ \\
\hline
\end{tabular}


$1,2, \ldots, 6)$ (vide a Tabela 3 ) podem ser observados na Fig. 14.

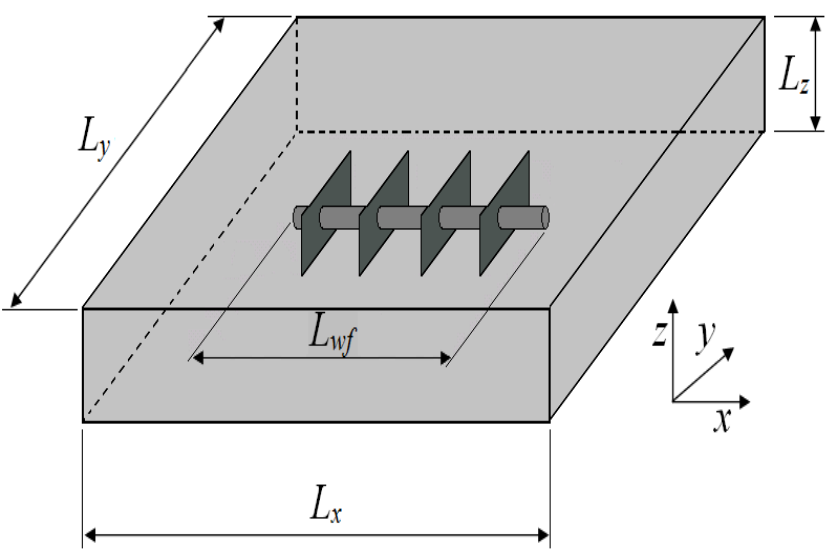

Figura 9: Representação de um poço horizontal com 4 fraturas.

Nas Figs. 10 e 11 pode-se notar claramente a influência da presença das fraturas em relação à simulação com um poço horizontal não fraturado. A queda de pressão do poço com fraturas foi menor do que a queda de pressão do poço sem fraturas, ou seja, um comportamento já esperado. Quanto menos a pressão no poço cair, uma vez que a resistência ao escoamento será menor nas fraturas, melhores são as condições em termos da preservação da energia natural do reservatório. Ele produzirá durante um tempo maior na recuperação primária, o que pode ser extremamente relevante na tomada de decisão sobre o uso futuro de técnicas de recuperação secundária.

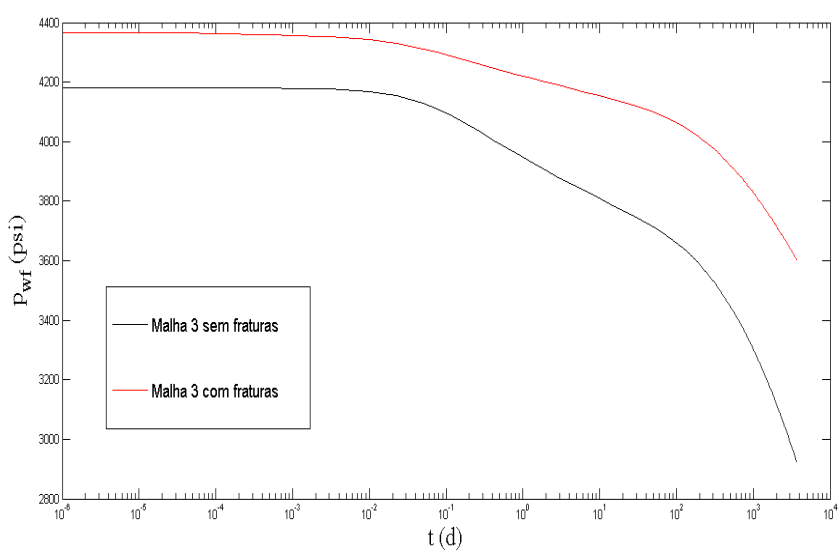

Figura 10: Resultado para o estudo de um poço horizontal com e sem fraturas (gráfico especializado).

Os campos de pressão para um período de 10 dias, considerando-se os casos do poço horizontal e o do poço horizontal hidraulicamente fraturado, podem ser vistos, respectivamente, nas Figs. 12 e 13. Os dois campos de pressão foram determinados para a camada central de células na direção $z$, na qual estão as fraturas hidráulicas. Pode-se notar o efeito das fraturas na mudança da forma da superfície de pressão, quando compara-se os

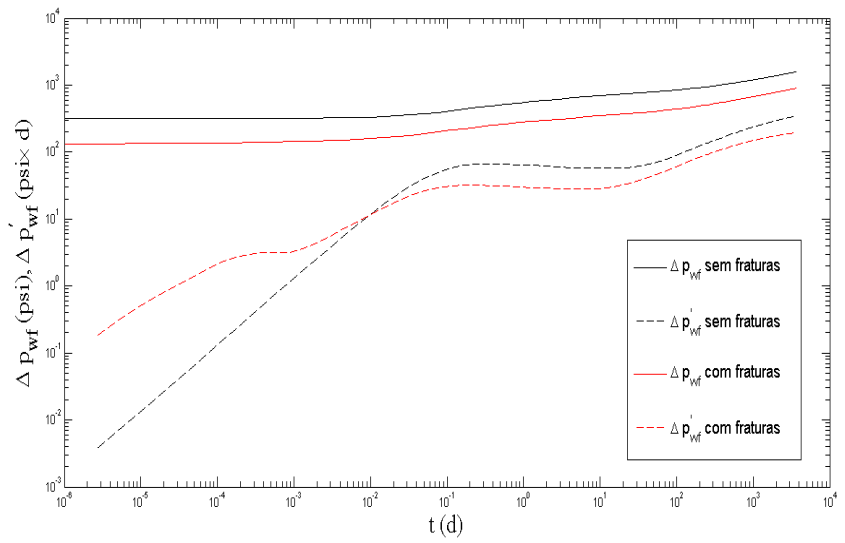

Figura 11: Resultado para o estudo de um poço horizontal com e sem fraturas (gráfico diagnóstico).

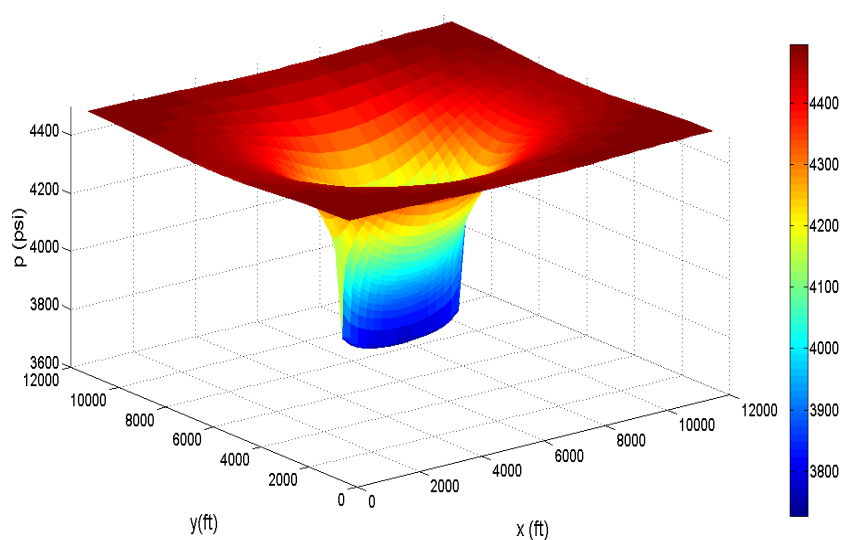

Figura 12: Resultado para o estudo de um poço horizontal sem fraturas.

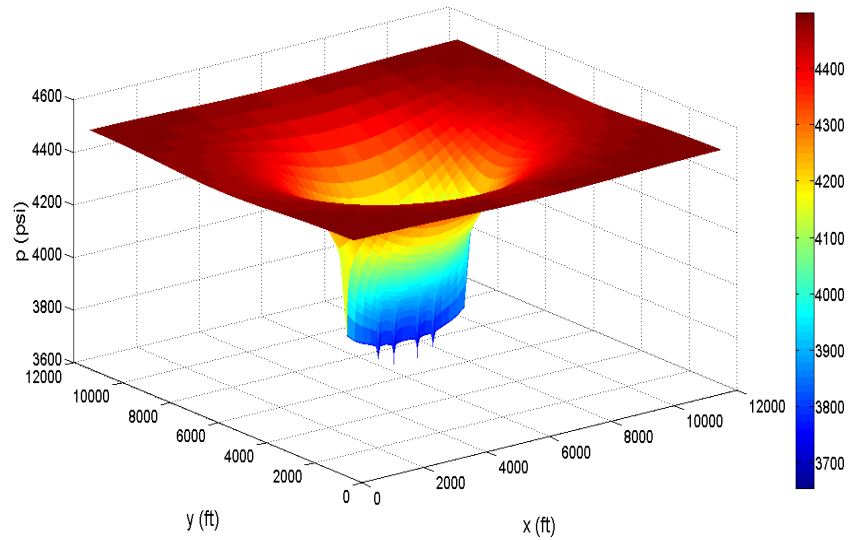

Figura 13: Resultado para o estudo de um poço horizontal com fraturas.

dois campos. Além disso, os valores numéricos são diferentes de forma que valores mínimos de pressão ocorrem nas fraturas (Fig. 13), mas as demais células possuem pressões mais elevadas do que no caso sem fraturamento, de forma que a energia do reservatório foi melhor preservada. 


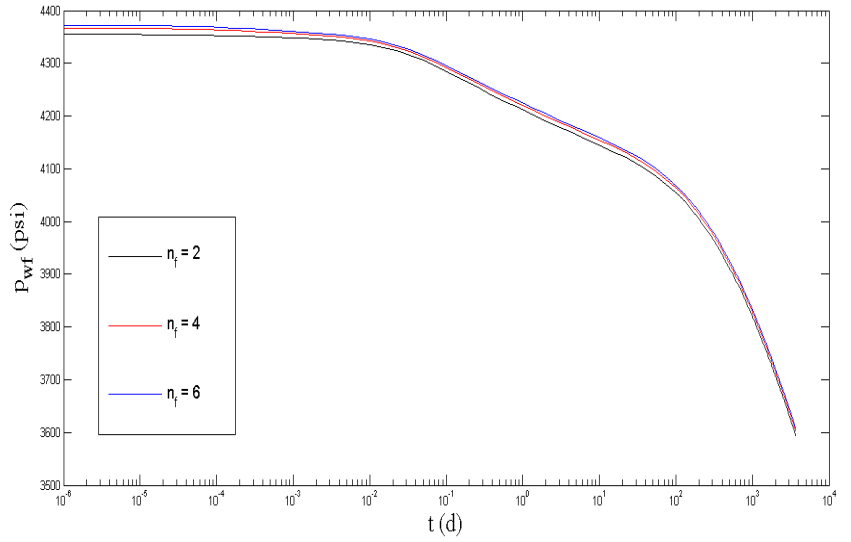

Figura 14: Resultado da variação de número de fraturas (gráfico especializado).

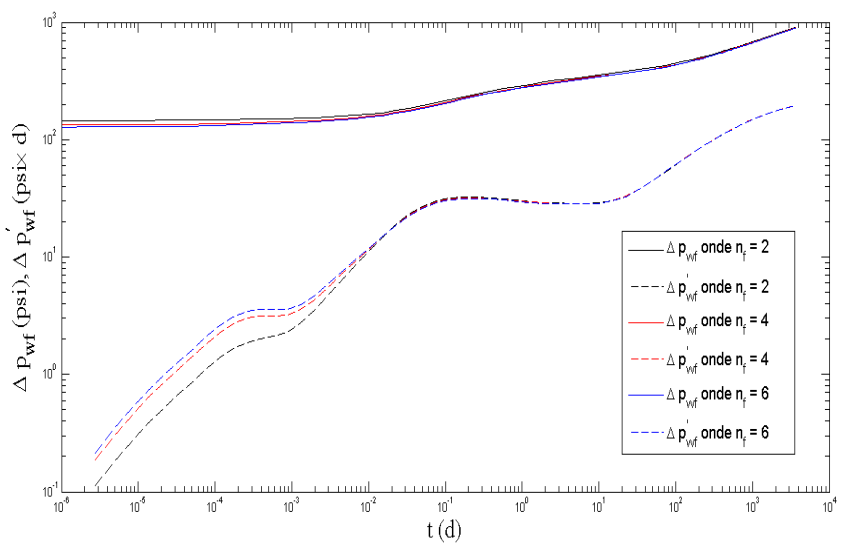

Figura 15: Resultado da variação de número de fraturas (gráfico diagnóstico).

Por se encontrarem na região do poço, a influência das fraturas ocorre no começo do escoamento e geram uma maior diferença nos resultados na região do artefato numérico, pois é quando começa a produção. A variação do número de fraturas, $n_{f}$, é importante porque quanto mais fraturas um poço tiver maior será a existência de caminhos preferenciais ao escoamento (vide as Figs. 14 e 15). Porém, não se pode desconsiderar o fato de que quanto mais fraturas um poço possuir, mais caro será o seu custo de produção.

Constata-se, a partir dos resultados já obtidos, que não foram identificados todos os regimes de escoamento presentes no trabalho de Wang et al. [24]. Primeiramente, deve-se destacar que o regime de estocagem no poço foi modelado nas soluções de Wang et al. [24], o que não foi feito nas simulações numéricas deste trabalho (onde há somente a presença de estocagem numérica associada ao artefato ligado ao modelo de acoplamento poço-reservatório). Além disso, é importante ressaltar que os regimes de escoamento previstos para um poço horizontal não ocorrem, necessariamente, para todos os sistemas poço-reservatório. De fato, na prática são poucos os casos nos quais há a presença de todos os regimes de escoamento e com uma identificação clara (às vezes al-

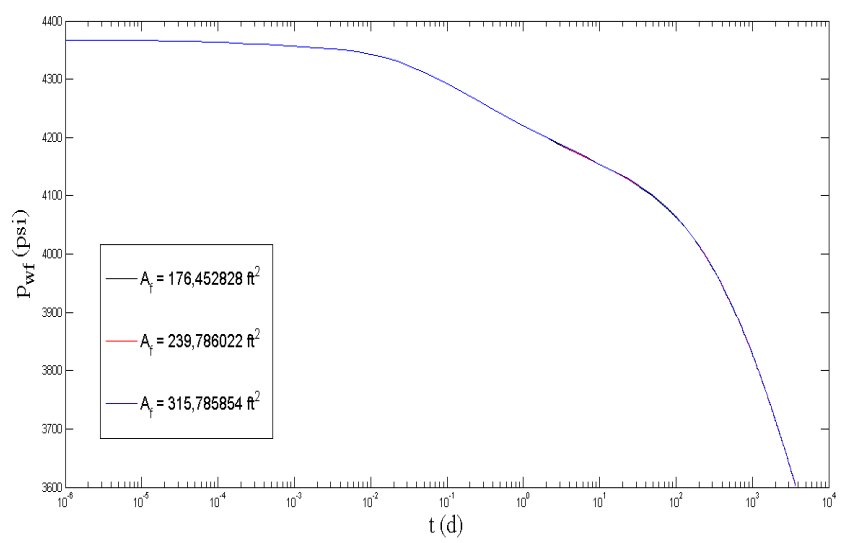

Figura 16: Resultado da variação da área da fratura (gráfico especializado).

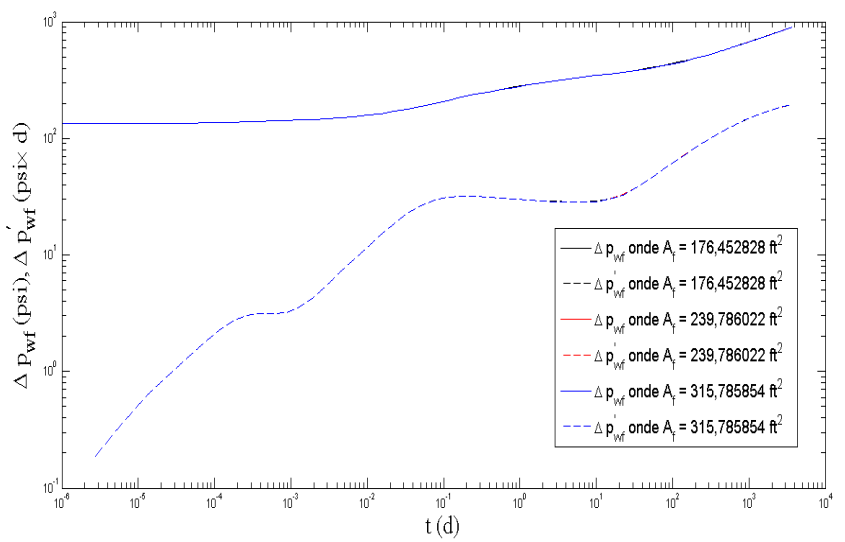

Figura 17: Resultado da variação da área da fratura (gráfico diagnóstico).

guns regimes ocorrem, mas em um período curto demais para que a sua representação no gráfico seja nítida).

Finalmente, deve-se notar que o tempo de produção precisa ser longo o bastante para que apareçam os efeitos de fronteira, que no caso de reservatórios de baixa permeabilidade portadores de óleo pode superar a escala de anos para que os regimes mais tardios estejam todos presentes.

A fim de se obter mais resultados, para a análise das fraturas em um poço horizontal, foram considerados diferentes valores para as propriedades das fraturas e da matriz (reservatório).

A variável estudada nos testes apresentados nas Figs. 16 e 17 foi a área da fratura, $A_{f}$. Houve alguma influência nos resultados em consequência da variação das áreas, porém o efeito não foi significativo para os valores testados.

Por outro lado, como pode ser observado nas Figs. 18 e 19, o comportamento dos resultados em função da variação da espessura da fratura, $h_{f}$, foi apreciável e percebido ao longo da simulação, com destaque para a região onde começa o escoamento (onde existe o artefato numérico).

Estudos foram realizados, mantida constante a razão de anisotropia, multiplicando-se os valores das permea- 


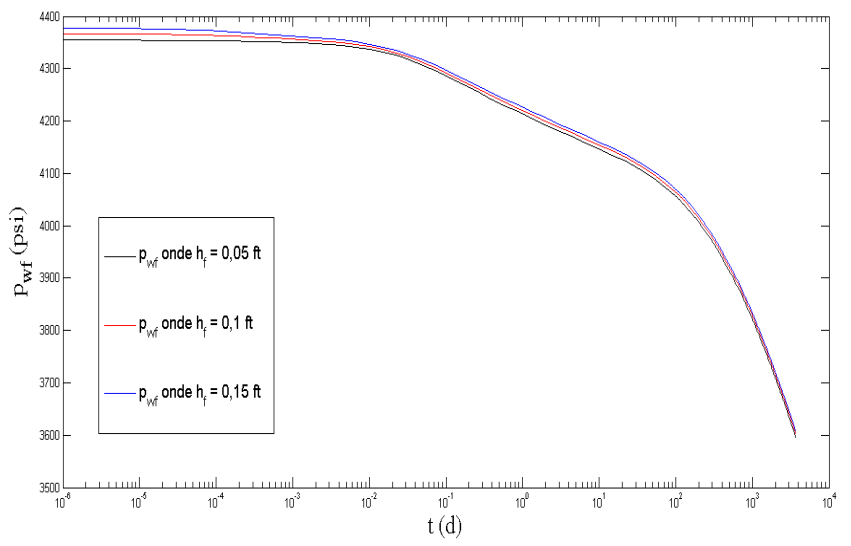

Figura 18: Resultado da variação da espessura da fratura (gráfico especializado).

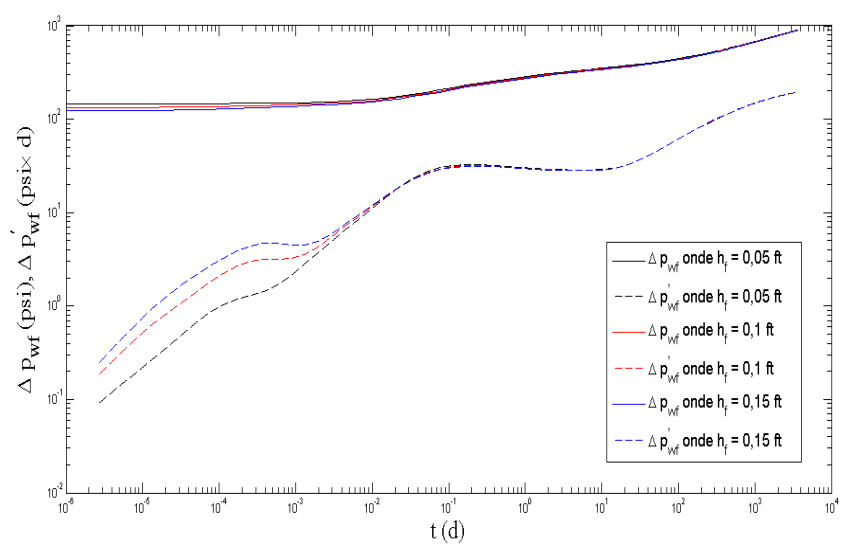

Figura 19: Resultado da variação da espessura da fratura (gráfico diagnóstico).

bilidades por um determinado fator. Para os testes com a variação da permeabilidade da matriz, $k_{m}$, mantevese o mesmo valor da permeabilidade da fratura, $k_{f}$. Em se tratando da variação da permeabilidade da fratura, a permeabilidade da matriz foi mantida fixa.

Sabendo-se que a permeabilidade mede a capacidade do fluido de escoar no meio poroso, pode-se observar pelos gráficos das Figs. 20 e 21 que há uma maior influência da fratura nos instantes iniciais da produção no poço.

Como pode ser visto nas Figs. 22 e 23, quanto maior a permeabilidade da matriz menos tempo dura o efeito do artefato numérico e menores são as quedas de pressão.

Agora, olhando-se para o gráfico da Fig. 23, fica clara a influência da permeabilidade da matriz ao longo de toda a simulação. Quanto maior a permeabilidade do reservatório, mais cedo ocorrem os efeitos da fronteira em decorrência do aumento na condutividade hidráulica do sistema $(C H S)$, dada por:

$$
C H S=\frac{k}{\phi \mu\left(c_{o}+c_{\phi}\right)} .
$$

Simulações variando os valores das porosidades da fratura e da matriz, $\phi_{f}$ e $\phi_{m}$ respectivamente, também foram realizadas. Com o emprego de diferentes valores para a porosidade da fratura observa-se (vide as Figs. 24

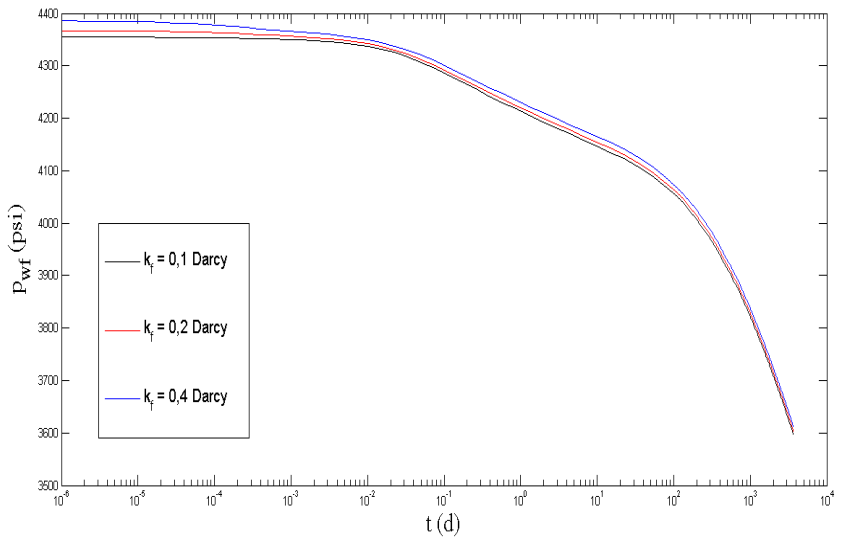

Figura 20: Resultado da variação da permeabilidade da fratura (gráfico especializado).

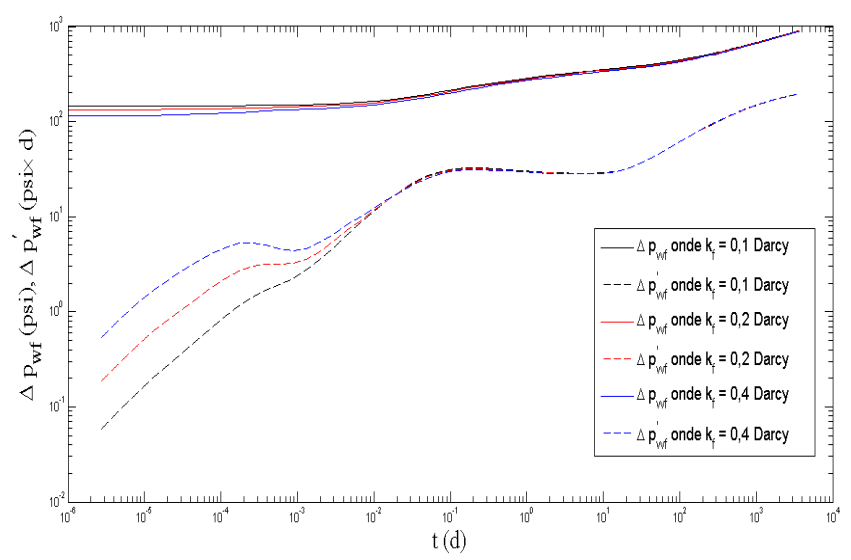

Figura 21: Resultado da variação da permeabilidade da fratura (gráfico diagnóstico).

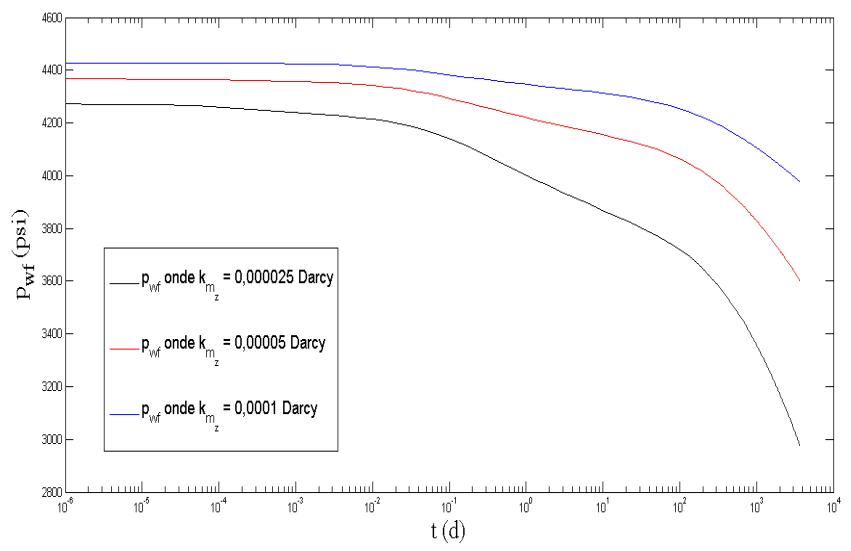

Figura 22: Resultado da variação da permeabilidade da matriz (gráfico especializado). 


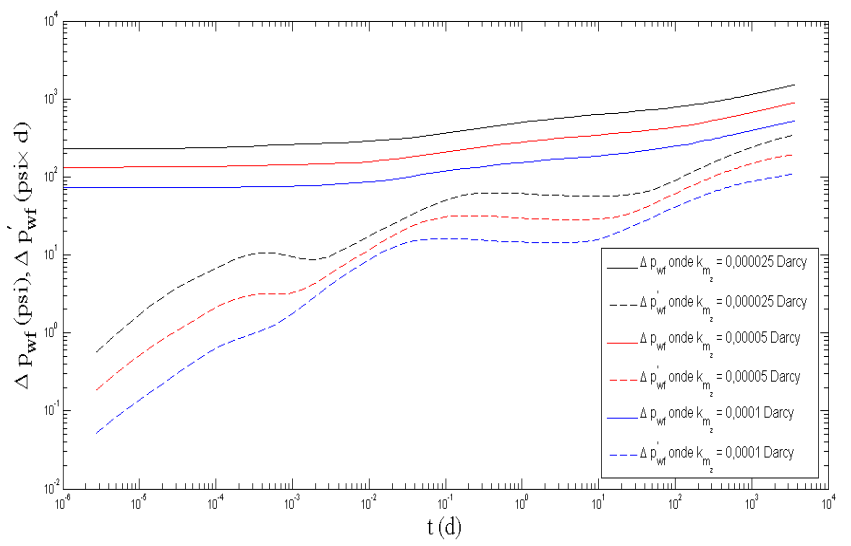

Figura 23: Resultado da variação da permeabilidade da matriz (gráfico diagnóstico).

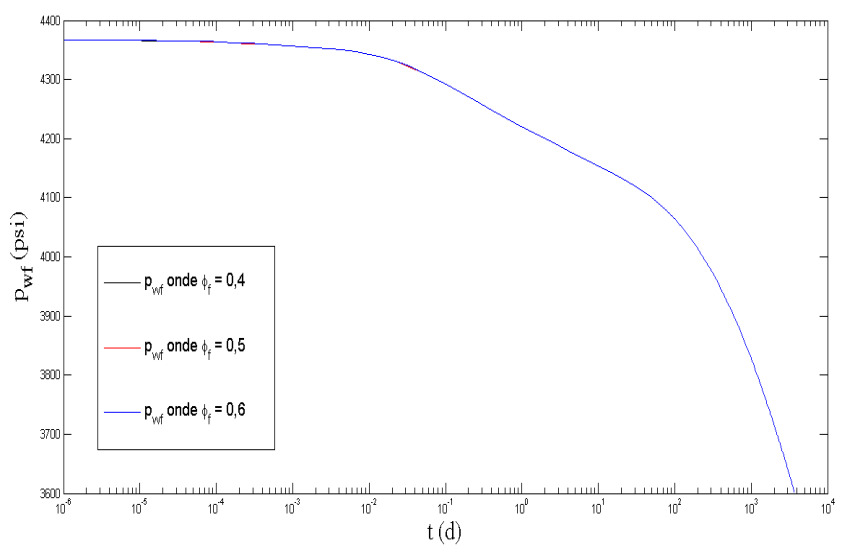

Figura 24: Resultado da variação da porosidade da fratura (gráfico especializado).

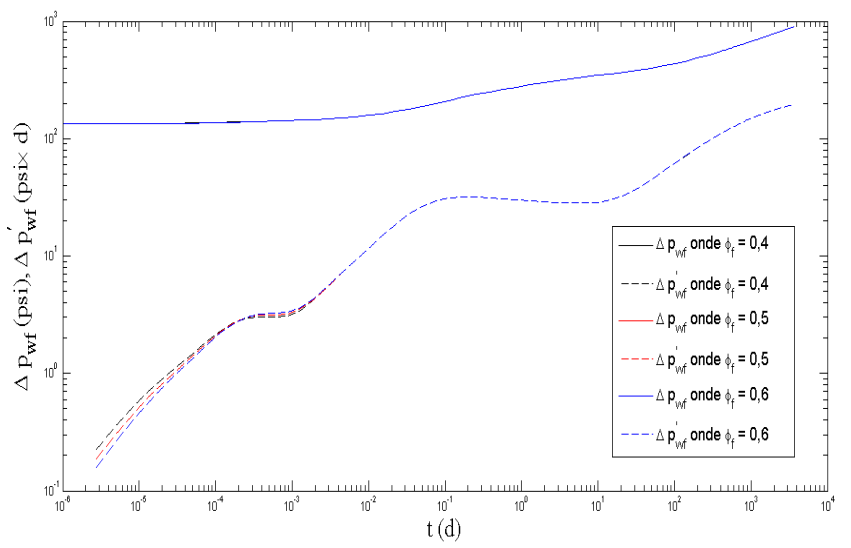

Figura 25: Resultado da variação da porosidade da fratura (gráfico diagnóstico).

e 25) que há uma maior influência desta propriedade na região onde também ocorre o artefato numérico. Para tempos curtos, as diferentes capacidades de armazenamento dadas pelas diferentes porosidades deixam de ter uma grande influência.

Nas Figs. 26 e 27, observa-se uma maior diferença no comportamento em quase toda a duração da simulação

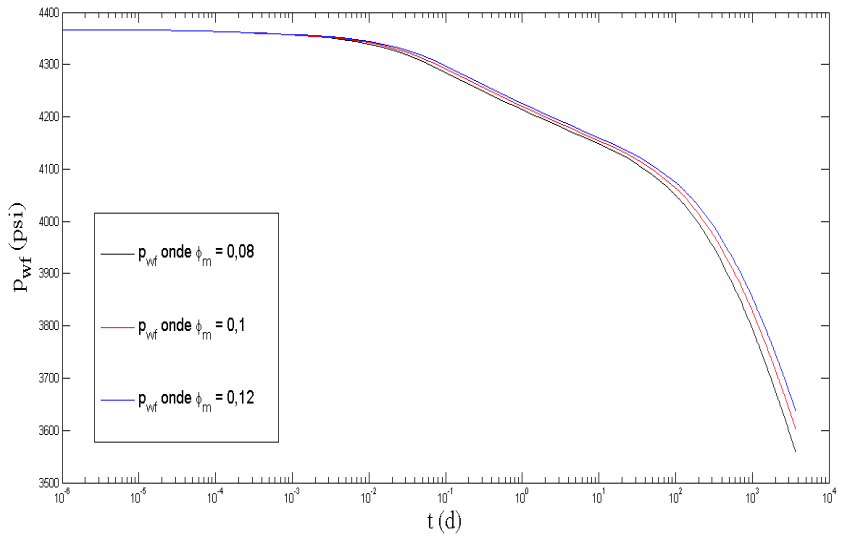

Figura 26: Resultado da variação da porosidade matriz (gráfico especializado).

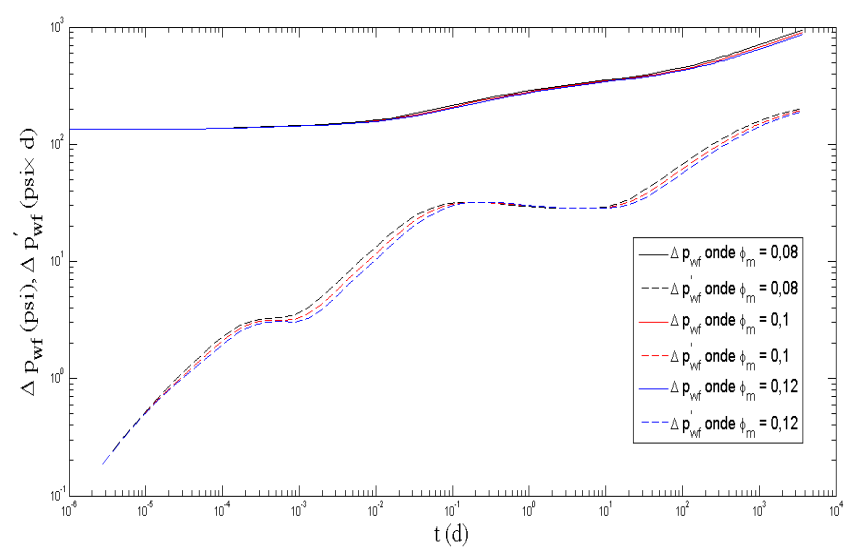

Figura 27: Resultado da variação da porosidade matriz (gráfico diagnóstico).

(alterou-se uma propriedade que diz respeito a todo o reservatório).

Três diferentes viscosidades do óleo foram utilizadas em mais um conjunto de simulações numéricas (Figs. 28 e 29). Quanto menor a viscosidade menor a duração do efeito do artefato numérico associado ao cálculo do raio equivalente. É notória a influência da viscosidade em toda a simulação.

Os resultados obtidos indicam que o simulador obteve resultados promissores para o estudo de reservatórios com poço horizontal hidraulicamente fraturado, em meios porosos representados em coordenadas cartesianas, para a técnica de solução numérica adotada neste trabalho, sendo que melhores resultados poderão ser determinados com a substituição futura da técnica de acoplamento poço-reservatório, eliminando-se o artefato numérico.

\section{Conclusões}

Neste trabalho estudou-se a produção de óleo leve, a partir de um reservatório de baixa permeabilidade, empregando-se um poço horizontal hidraulicamente fraturado. Um simulador numérico de reservatórios, apro- 


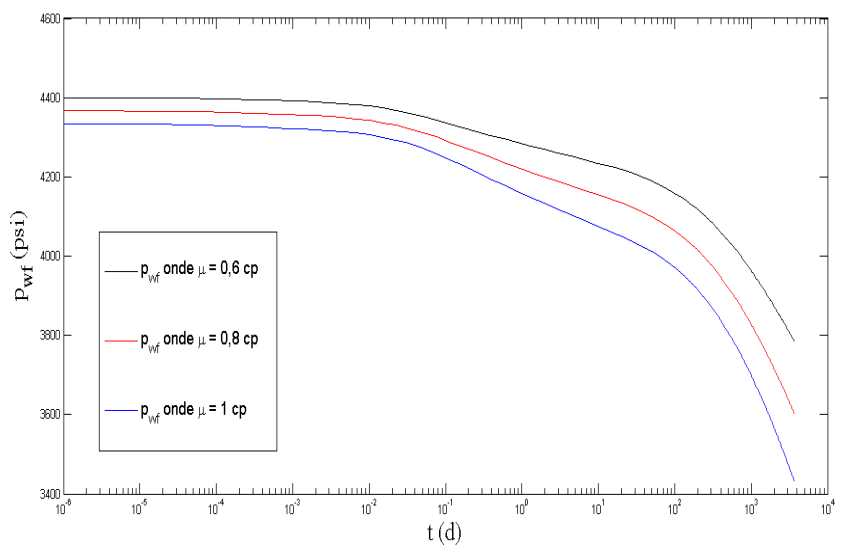

Figura 28: Resultado da variação da viscosidade do óleo (gráfico especializado).

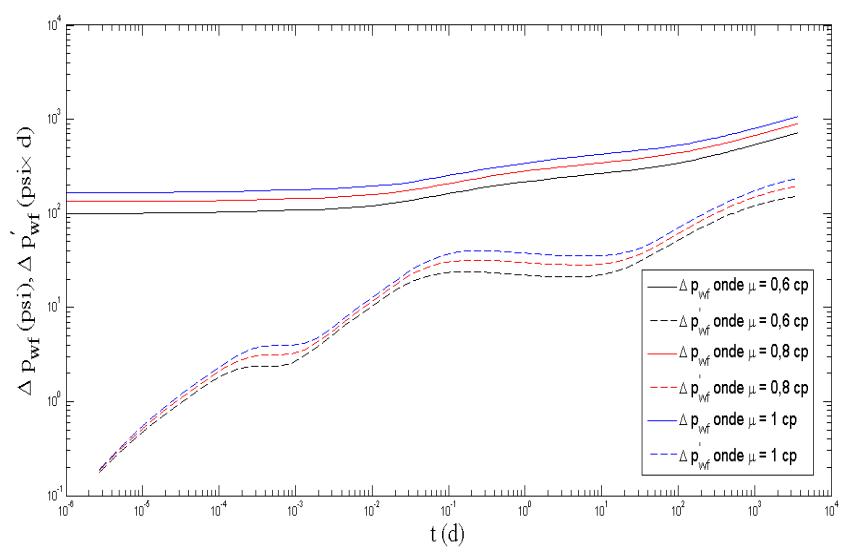

Figura 29: Resultado da variação da viscosidade do óleo (gráfico diagnóstico).

priado para aplicações na escala de campo, empregando o sistema de coordenadas cartesianas e uma abordagem clássica do tipo diferenças finitas, foi utilizado nesse estudo. Anteriormente, este simulador e suas opções de refinamento de malha tinham sido aplicadas apenas para escoamentos em reservatórios naturalmente fraturados com poços verticais. A equação diferencial parcial nãolinear resultante do processo de discretização, escrita em termos da pressão, foi resolvida numericamente, após linearização, usando o método iterativo dos Gradientes Conjugados Pré-condicionado. O objetivo principal deste trabalho foi o estudo dos efeitos combinados das fraturas hidráulicas com o poço horizontal, considerando-se diferentes cenários de produção de óleo leve, no perfil da perda de pressão no poço produtor.

Deve-se ressaltar que o estudo da simulação numérica do sistema de produção de óleo leve em formações de baixa permeabilidade, ou Light Tight Oil, merece destaque no contexto de reservas não convencionais. De fato, mesmo nos dias atuais, o mundo ainda possui uma dependência com relação ao uso dos combustíveis fósseis, principalmente do petróleo. Como este cenário ainda perdurará nas próximas décadas, ao que tudo indica, tornase importante o desenvolvimento de novas técnicas de exploração e de produção de hidrocarbonetos, de modo que o processo possa ser otimizado. Os reservatórios convencionais que são considerados reservatórios de "fácil produção" não estavam atendendo, até pouco tempo atrás, à demanda energética mundial, fazendo-se necessário a explotação de reservas não convencionais que são caracterizadas por sua baixas permeabilidade e porosidade ou alta viscosidade.

Os resultados obtidos destacam a influência do artefato numérico nos tempos iniciais, quando também são importantes os efeitos das fraturas hidráulicas no acoplamento poço-reservatório. Pelo que pôde ser observado, o tempo de ocorrência do artefato e a sua magnitude são funções da permeabilidade, sendo que quanto menor for a permeabilidade (e para uma maior viscosidade) maior será a sua duração e magnitude. Os resultados comprovaram a viabilidade da técnica do fraturamento hidráulico, no sentido de reduzir-se a queda de pressão no reservatório (preservando, desta forma, a energia natural da jazida). Esse fato é relevante na definição de quando se deve aplicar os métodos de recuperação secundária. Além disso, em alguns casos, vazões minimamente viáveis economicamente só são atingidas através do fraturamento hidráulico. Ressalta-se que há uma análise de custo-benefício a ser feita, na qual as simulações numéricas são de grande valia: discutir o custo de se obter um dado número de fraturas com dadas permeabilidade e porosidade, em relação ao quanto de energia será preservada. Neste sentido, a distribuição das fraturas ao longo do poço horizontal também é um parâmetro relevante a ser considerado e estudado.

\section{Agradecimentos}

Os autores gostariam de agradecer o suporte financeiro fornecido pelo CNPq.

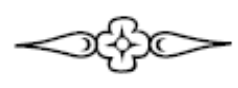

\section{Referências}

[1] Bourdet, D. (2002). Well Test Analysis: the Use of Advanced Interpretation Models. Handbook of Petroleum Exploration and Production 3. Elsevier, Amsterdam.

[2] Tan, T. (2011). Bakken Shale Oil Production Trends. Dissertação de mestrado, Texas A\& M University, Texas.

[3] Dongyan, F., Yao, J., Sun, H., Zeng, H., and Wang, W. (2015). A composite model of hydraulic fractured horizontal well with stimulated reservoir volume in tight oil \& gas reservoir. Journal of Natural Gas Science and Engineering, 115-123.

[4] Ali, J. A., Siddiqui, S., and Dehghanpour, H. (2013). Analyzing the production data of fractured horizontal wells by a linear triple porosity model: Development of analysis equations. Journal of Petroleum Science and Engineering, 117-128. 
[5] Wang, H., Liao, X., Lu, N., Cai, Z., Liao, C., and Dou, X. (2013). A study on development effect of horizontal well with srv in unconventional tight oil reservoir. Journal of the Energy Institute, 117-128.

[6] Chaohua, G., X., J., W., M., and Ruizhong, J. (2015). Experimental study and numerical simulation of hydraulic fracturing tight sandstone reservoirs. Elsevier, 334344 .

[7] Zhang, K. (2014). Potential Technical Solutions to Recover Tight Oil. Dissertação de mestrado, Norwegian University of Science and Technology, Calgary.

[8] Wendong, W., Shahvali, M., and Yuliang, S. (2015). A semi-analytical fractal model for production from tight oil reservoirs with hydraulically fractured horizontal wells. Fuel - Elsevier, 158, 613-618.

[9] Zhao, L. (1994). Horizontal Well Simulation with Local Grid Refinement. Ph.D. thesis, Texas A \& M University.

[10] Prado, R. P. S. (2003). Comparação Entre Estratégias de Produção Utilizando Poços Verticais e Horizontais. Dissertação de mestrado, Universidade Estadual de Campinas, Campinas.

[11] Bessa Jr., F. P. (2014). Análise da Recuperação em Reservatórios de Gás com Baixa Permeabilidade (Tight Gas) Através do Fraturamento Hidráulico. Dissertação de mestrado, Universidade Federal do Rio Grande do Norte, Natal.

[12] Raghavan, R. S., Chen, C. C., and Agarwal, B. (1994). Analysis of horizontal wells intercepted by multiple fractures. In Permian Basin Oil and Gas Recovery Conference, 1994, Midland, TX. Proceedings of the Permian Basin Oil and Gas Recovery Conference, Midland, TX: SPE.

[13] Valko, P. and Economides, M. J. (1996). Performance of fractured horizontal wells in high-permeability reservoirs. In Paper SPE 31149 presented at the Society of Petroleum Engineers International Symposium Annual on Formation Damage Control, Lafayette, LA.

[14] Bello, R. O. and Wattenbarger, R. (2008). Rate transient analysis in naturally fractured shale gas reservoirs. In Gas Technology Symposium Joint Conference, 2008, Calgary, Canada. CIPC/SPE Annual Gas Technology Symposium Joint Conference., Calgary, Canada: CIPC/SPE.

[15] Thomas, J. E. (2004). Fundamentos de Engenharia de Petróleo. Handbook of Petroleum Exploration and Production 3. Interciência, Rio de Janeiro.

[16] Ertekin, T., Abou-Kassem, J. H., and King, G. R. (2001). Basic Applied Reservoir Simulation. SPE Textbook Series 7. Society of Petroleum Engineers, Richardson.

[17] Rosa, A. J., Carvalho, R. S., and Xavier, J. A. D. (2006). Engenharia de Reservatórios de Petróleo. Interciência, Rio de Janeiro, Brasil.

[18] Dandekar, A. Y. (2013). Petroleum Reservoir Rock and Fluid Properties. CRC Press, USA.

[19] Tiab, D. and Donaldson, E. C. (2004). Petrophysics, Theory and Practice of Measuring Reservoir Rock and Fluid Transport Properties. Gulf Professional Publishing, Burlington, USA, second edition.
[20] Dumkwu, F. A., Islam, A. W., and Carlon, E. S. (2012). Review of well models and assessment of their impacts on numerical reservoir simulation performance. Journal of Petroleum Science and Engineering, (82-83), 174-186.

[21] Bourdet, D., Ayoub, J. A., and Pirard, Y. M. (1989). Use of pressure derivative in well-test interpretation. SPE Formation Evaluation, 293-302.

[22] Bourdet, D. P., Whittle, T. M., Douglas, A. A., and Pirard, Y. M. (1983). A new set of type cruves simplifies well test analysis. World Oil, 95-106.

[23] Camargo, C. (1993). Comportamento Transiente de Pressão em Poços Horizontais. Dissertação de mestrado, Universidade Estadual de Campinas, Campinas.

[24] Wang, H., Liao, X., and Zhao, X. (2014). Study of tight oil reservoir flow regimes in different treated horizontal well. Journal of the Energy Institute, 200-201.

[25] Amaral Souto, H. P. and de Souza, G. (2014). Numerical reservoir simulation of naturally fractured reservoirs. In Ibero-Latin American Congress on Computational Methods in Engineering, 35., 2014, Fortaleza. Proceedings of the XXXV Ibero-Latin American Congress on Computational Methods in Engineering, Fortaleza.

[26] Nick, H. M. et al. (2013). Reactive dispersive contaminant transport in coastal aquifers: Numerical simulation of a reactive henry problem. Journal of Contaminant Hydrology, 145, 90-104.

[27] Al-Mohannadi, N. (2004). Simulation of Horizontal Well Tests. Ph.D. thesis, Colorado School of Mines, Golden, USA.

[28] Peaceman, D. W. (1983). Interpretation of well-block pressures in numerical reservoir simulation with nonsquare grid blocks and anisotropic permeability. Society of Petroleum Engineers Journal, 23(3), 531-543. 